\title{
INTERESSI MILITARI NELLE ELLENICHE DI OSSIRINCO
}

\author{
ALESSANDRO BRAMBILLA (*)
}

Nota presentata dal m.e. Cinzia Bearzot

(Adunanza del 21 dicembre 2017)

SunTO. - Il contributo prende le mosse dal tentativo di definire cosa sia uno storico militare e di stabilire se tale definizione sia applicabile, come fatto da alcuni studiosi, all'autore delle Elleniche di Ossirinco. Partendo da un'analisi degli eventi bellici descritti dall'autore, viene rimarcata la cura di quest'ultimo nel descrivere gli spostamenti delle truppe nel corso di una campagna, nell'annotare il numero di caduti e l'interesse verso alcuni aspetti tattici, come stratagemmi e imboscate, ed economici, ad esempio relativi al pagamento dei soldati. Al contempo si sottolinea che la precisione dimostrata nel descrivere certi episodi, se da un lato dimostra che l'autore poteva contare su una documentazione di buona qualità, forse resoconti ufficiali, da sola non è un elemento sufficiente per permettere di qualificare le Elleniche di Ossirinco come un'opera di uno storico militare.

$* * *$

ABSTRACT. - This paper starts from the attempt to define what is a "military historian" and tries to establish whether this definition is applicable to the author of the Hellenica Oxyrhynxhia. Moving from the analysis of the military related episodes described by the author, the paper underlines his care in relating the movements of troops throughout a campaign, in recording casualties, and his attention towards tactics, such as stratagems and abushes, or economic matters, such as the payment of troops. At the same time, this paper emphasises that the precision showed by the author in describing these warfare episodes, though possibly demonstrating the employment of good quality sources, maybe official accounts, it is not enough to define the Hellenica Oxyrbynchia as the work of a military historian.

(") Dipartimento di Storia, archeologia e storia dell'arte, Facoltà di Lettere e Filosofia, Università Cattolica del Sacro Cuore, Milano, Italia.

E-mail: alexbrambilla86@gmail.com 


\section{IL QUADRO GENERALE}

Sin dalla scoperta dei frammenti papiracei che ne riportano il testo, le cosiddette Elleniche di Ossirinco non hanno mancato di suscitare un forte interesse tra gli studiosi. Accanto al dibattito ancora non archiviato circa l'identità dell'autore ${ }^{1}$ e la data di composizione dell'opera $^{2}$, molti contributi hanno preso in esame la tradizione storiografica e i problemi concreti derivanti dallo stato lacunoso in cui è giunto il testo. Inoltre, non sono affatto pochi gli studi che si sono concentrati sull'analisi approfondita di alcuni aspetti specifici del testo, come ad esempio gli interessi politico-istituzionali dell'autore ${ }^{3}$ e la sua curiosità nel descrivere le caratteristiche del funzionamento di alcune realtà costituzionali greche, nello specifico quella della lega beotica. ${ }^{4}$

Nelle parti di testo giunte fino a noi e delle quali riusciamo a leggere o a ricostruire in linea di massima il contenuto è possibile trovare descritte svariate tipologie di quelli che potremmo chiamare in maniera generica eventi violenti, come battaglie terrestri e navali, assedi, incursioni contro città o insediamenti di dimensioni più ridotte, azioni di saccheggio, imboscate, occupazioni territoriali, ma anche episodi di guerra civile, insurrezioni di città alleate contro la potenza egemone, nello specifico Atene, o ribellioni di soldati all'interno di un esercito. In totale è possibile isolare almeno ventisette di queste situazioni.

Nei frammenti sopravvissuti dell'opera siamo in grado di leggere una descrizione sufficientemente ampia di sole due battaglie terrestri, ovvero quella combattuta da Ateniesi e Megaresi sul territorio di questi ultimi presso Nisea nel $409^{5}$ e la vittoria dell'esercito comandato da

1 Per un'analisi dettagliata delle principali posizioni a riguardo, cfr. Lérida Lafarga 2007, pp. 114-206.

2 Lérida Lafarga 2007, 207-267.

3 Bearzot 1985; Bruce 1962.

4 Bonner 1910; Lanzillotta 2001.

5 Hell. Oxy. 1. Per la divisione in capitoli dell'opera, si è seguito il criterio adottato da McKechnie - Kern 1988. Tutte le date sono da intendersi a.C., salvo diversa indicazione.

6 Hell. Oxy. 11.3-6.

7 Hell. Oxy. 4. Sulle differenze tra la narrazione della battaglia contenuta nelle 
Agesilao presso Sardi nel 3956. Per quanto riguarda gli scontri navali, sono quattro gli episodi riportati. Il primo è la battaglia di Nozio del $406^{7}$. Segue lo scontro tra una flotta comandata da Timolao di Corinto e la flotta ateniese guidata da un certo Simico, narrato in una digressione del testo nella quale vengono riportati gli eventi dell'anno $411 / 10^{8}$. Viene inoltre descritta una schermaglia che vide coinvolte una trireme comandata dall'ateniese Demeneto, una seconda trireme comandata dallo spartano Milone e una terza nave, forse appartenente a una città menzionata poco prima, in una parte di testo che non ci è giunta'. Infine, per quanto riguarda gli eventi presentati nel frammentario terzo paragrafo del nono capitolo, è possibile che in questa sede venisse descritto il blocco navale portato dalla flotta di Conone a Cauno, sempre nell'anno 397/6, anche se il cattivo stato di conservazione del testo non permette di prendere posizione in maniera risolutiva a riguardo ${ }^{10}$.

L'unico riferimento a un vero e proprio assedio è piuttosto indiretto, dal momento che l'autore descrive uno scambio di messaggi fra una sentinella ateniese sulle mura di una città assediata, il cui nome non ci è noto, e un uomo originario di Mindo ${ }^{11}$. Al contempo, vi sono cinque accenni a incursioni compiute contro città o insediamenti minori. Tre di questi assalti, nello specifico contro Leontos Kephalai, Gordio ${ }^{12}$ e Miletou Teichos, furono compiuti dall'esercito di Agesilao nel suo percorso in Asia Minore nel 395 e tutti e tre fallirono ${ }^{13}$. Vi è poi la menzione di un infruttuoso attacco ateniese alla città di Efeso condotto da Trasillo nel $409^{14}$ e la descrizione del raid tebano contro la città di Iampoli nel $395^{15}$. Più frequenti sono invece i cenni a episodi di saccheggio, ancora una volta perpetrati principalmente dall'esercito al

Elleniche di Ossirinco e quella presente nella Vita di Alcibiade plutarchea, cfr. Mossé 2001.

8 Hell. Oxy. 7, 4.

9 Hell. Oxy. 8, 1-2.

10 Hell. Oxy. 9, 3.

11 Hell. Oxy. 5.

12 Hell. Oxy. 21, 5-6.

13 Hell. Oxy. 22, 3.

14 Si tratta di un riferimento contenuto nel papiro del Cairo; cfr. McKechnie Kern 1988, 116-121.

15 Hell. Oxy. 18, 5.

16 Hell. Oxy. 12, 1-2 e 21, 3-5.

17 Hell. Oxy. 21, 1. 
comando di Agesilao in Asia Minore, soprattutto in Frigia ${ }^{16}$ e Misia ${ }^{17}$, ma anche dagli uomini di Timolao di Corinto contro alcune isole che erano schierate dalla parte degli Ateniesi ${ }^{18}$ e dai Tebani contro alcuni centri al confine tra Focide e Beozia nel $395^{19}$.

Nel capitolo ventunesimo, secondo paragrafo, vengono narrate due imboscate strettamente connesse fra di loro: mentre l'esercito di Agesilao era impegnato nella marcia attraverso il territorio della Misia, la retroguardia fu attaccata da alcuni soldati locali nei pressi del Monte Olimpo, lasciando sul terreno una cinquantina di uomini. In risposta, il re spartano ordinò ai suoi uomini di compiere una manovra diversiva, che condusse i Misi impegnati nell'attacco a cadere in trappola, perdendo più di centotrenta combattenti $i^{20}$.

Infine, nel testo delle Elleniche di Ossirinco giunto fino noi è possibile leggere la descrizione di altri episodi che implicarono l'uso della forza, ossia la ribellione di Taso, sostenuta da Timolao di Corinto, contro il dominio despotico di Atene nel $411^{21}$, il colpo di stato che ebbe luogo a Rodi nel 395, che vide il diretto coinvolgimento di Conone ${ }^{22}$, e la rivolta di una parte delle truppe dello stesso Conone a Cauno e Rodi nel medesimo anno, causata dal mancato pagamento di quanto dovuto ai soldati ${ }^{23}$.

Si può notare, dunque, come, nonostante lo stato lacunoso dell'opera, non siano pochi i riferimenti più o meno ampi a eventi bellici, tanto che alcuni moderni hanno lodato l'abilità dell'autore nel raccontare questi episodi. Iain Bruce, ad esempio ne sottolineava la particolare attenzione ai dettagli, soprattutto per quanto riguarda i movimenti dell'esercito di Agesilao in Persia, mentre Paul McKechnie e Stephen Kern, nel loro commento all'opera, ponevano l'accento sul fatto che nelle Elleniche di

18 Hell. Oxy. 7.4. Questa prevalenza di riferimenti ad Agesilao in Asia Minore e ai Tebani in Focide nel contesto della non sorprende, dal momento che queste sono le uniche due spedizioni, ovvero operazioni militari più strutturate e di lunga durata, che vengono descritte con una certa ampiezza nei frammenti delle Elleniche in nostro possesso.

19 Hell. Oxy. 18, 2-3.

20 Hell. Oxy. 21, 2.

21 Hell. Oxy. 7, 4.

22 Hell. Oxy. 15.

23 Hell. Oxy. 20. 
Ossirinco è individuabile uno specifico interesse per gli stratagemmi e per le imboscate. Paul Cartledge si è spinto fino al punto di dire che l'autore dell'opera agisce come un abile storico militare ${ }^{24}$.

Ciononostante, queste osservazioni da parte dei moderni sono piuttosto occasionali e manca una valutazione complessiva dell'approccio dimostrato nelle Elleniche per quanto riguarda gli interessi militari.

\section{UNO STORICO MILITARE?}

Prima di concentrare l'attenzione sulla maniera in cui l'opera presenta i diversi episodi bellici dei quali abbiamo già fatto menzione, è opportuno domandarsi cosa sia uno "storico militare" e, in secondo luogo, quali elementi permettano di definire valido e capace uno storico militare.

Per quanto si tratti di una branca di studi decisamente antica, non è per niente facile tracciare con precisione i limiti del campo di studi di tale disciplina. Una prima definizione intuitiva può essere la seguente: lo storico militare è uno studioso che si occupa di studiare le guerre, le battaglie, gli eserciti e gli armamentii ${ }^{25}$. Tuttavia, questa definizione così netta rischia di essere ambigua, se non addirittura poco accurata. Certamente, al centro degli studi di storia militare ci sono le storie dei conflitti, sia di specifiche guerre, sia della condotta delle operazioni militari nel senso più ampio. Al contempo, però, la narrazione di campagne e scontri e l'esame dei meccanismi e dei principi del fare la guer$\mathrm{ra}$, ovvero la storia delle tattiche, non esaurisce il campo della disciplina. L'organizzazione degli eserciti, come essi venivano reclutati e mobilitati, in quale maniera combattevano dal punto di vista tecnico, con quali armi, con quali strategie, sono solo una parte dell'indagine di cui si fa carico uno studioso di storia militare. Le implicazioni sociali ed economiche della guerra, lo studio delle istituzioni militari, dell'evoluzione tecnologica, l'analisi dei cambiamenti nei valori che definiscono il combattente e dell'esperienza del singolo in battaglia, nonché gli aspetti culturali implicati dal fenomeno sono ugualmente tematiche che vengo-

24 Cartledge 1987, 66. Per una panoramica riguardante i diversi giudizi espresso dai moderni sull'opera, cfr. Occhipinti 2016, 1-11.

25 Cfr. ad esempio Howard et al. 1988. 
no normalmente prese in esame dagli storici militari.

Si giunge, quindi, a una definizione di storia militare che comprende non solo la storia di singole e battaglie, ma qualunque studio storico in cui l'attenzione sia rivolta anche all'astrazione teorica sulla maniera in cui si combatteva per mare e per terra, alle istituzioni militari, alle intersezioni tra il fenomeno della guerra e la politica, l'economia, la società, la natura, la cultura e così via ${ }^{26}$.

Un'ovvia conseguenza di una definizione ampia è che molte opere di storia militare possono al contempo essere classificate anche come opere di storia politica, economica, istituzionale, del pensiero, sociale e culturale, il che dimostra come spesso queste categorie siano costrutti astratti e artificiali, dai contorni porosi e permeabili, cosa ancor più vera per quanto riguarda le opere storiografiche antiche. Di conseguenza, uno storico militare può essere anche classificato come storico della politica, storico dell'economia, storico della società e così via, in base alla prospettiva adottata in maniera prevalente nella sua opera.

A questo punto risulta ancora più complesso definire cosa si intenda con la qualifica di storico militare capace e valido o, per dirla con Cartledge, good military bistorian.

Innanzitutto, e forse è superfluo ricordarlo, essere uno storico militare non è solo e solamente una questione di curriculum, ovvero non si lega necessariamente all'esperienza personale di guerra, come invece accadeva fino alla prima metà del Novecento. Oggi è possibile raggruppare coloro che si occupano di studi di storia militare in tre grandi categorie: accademici di professione, scrittori dilettanti e specialisti militari con esperienza sul campo, ovvero soldati o ufficiali in attività o in congedo. Il fatto che l'autore di uno studio di storia militare non sia un accademico non significa ipso facto che l'opera non possa essere un valido contributo. Al contempo, abbiamo molti esempi di volumi scritti da storici di professione o da ex soldati che sono a malapena leggibili.

L'etichetta di abile storico militare ha certamente a che fare con il contenuto dell'opera e con la maniera in cui esso è presentato dall'autore. Una semplice narrazione evenemenziale o l'accostamento delle fonti senza alcun tentativo di capire che cosa queste ultime ci dicono

26 Cfr. Morillo - Pavkovic 2006, 1-4. Si vedano anche Black 2004, 17-18; Citino 2007, 1070-1090. 
nel contesto più ampio degli studi sull'uomo rischia di diventare un mero esercizio antiquario. Al contrario, il compito di ogni storico è quello di analizzare le fonti, cercando di capire cosa ci trasmettono su tematiche più ampie e trasversali. Pertanto, gli storici militari non possono limitarsi alla semplice narrazione degli eventi, ma devono affiancare un'approfondita analisi di temi specifici e una ricca sintesi che apra a prospettive più ampie, e questo è ormai un concetto acquisito.

Ad ogni modo vi è un ulteriore elemento che permette di definire valido e capace uno storico militare, e questo ha a che fare con la maniera in cui lo studioso si avvicina alla materia, il che ci riconduce alle Elleniche di Ossirinco.

Nel descrivere le fonti a disposizione per ricostruire la vita di Agesilao, Paul Cartledge mette a confronto la maniera di operare dell'anonimo autore che stiamo prendendo in considerazione con quella di Senofonte in questi termini:

While Xenophon was clearly concerned primarily with the personality of Agesilaos and so concentrated on episodes that lent themselves to more picturesque or dramatic treatment, $\mathrm{P}$ as a good military historian sturdily ignored the incidentals retailed by Xenophon and allowed the reader to see clearly the truth Xenophon strove to obfuscate, namely that Agesilaos achieved no notable victories. P's flat, "antirhetorical" style was an admirable vehicle for this approach ${ }^{27}$.

Di certo un tale giudizio non è lusinghiero nei confronti di Senofonte.

Ora, Cartledge descrive l'autore delle Elleniche di Ossirinco come un abile storico miliare, e implicitamente Senofonte come uno storico militare scadente, sulla base del fatto che il primo non ha secondi fini, ossia uno scopo personale che invece caratterizzerebbe l'opera del secondo, ovvero il tentativo di nascondere le colpe di Agesilao: l'anonimo è uno storico militare valido perché si rifiuta di narrare quegli aspetti marginali e quegli elementi solo vagamente connessi con la vicenda presa in esame tanto cari a Senofonte, concentrandosi solamente sulle informazioni che risultano rilevanti per fornire al lettore l'occasione di

27 Cartledge 1987, 66. 
comprendere in maniera adeguata cosa sia successo sul campo di battaglia, e questo adottando uno stile per nulla retorico, permettendo al lettore di avvicinarsi il più possibile alla realtà dei fatti. militare:

Pertanto, seguendo l'affermazione di Cartledge, un abile storico

1) non ha un'agenda personale, dei secondi fini nella narrazione, o perlomeno non permette alle proprie opinioni e ai propri sentimenti di pregiudicare la possibilità di offrire al lettore una ricostruzione degli eventi quanto più possibile oggettiva;

2) non dissemina il suo racconto di dettagli minori e incidentali, ma offre solo informazioni rilevanti;

3) adotta uno stile lineare e semplice, rifiutando gli artifici retorici.

È necessario allora domandarsi se l'autore delle Elleniche di Ossirinco corrisponda realmente a questa definizione. Per fare ciò, prenderemo in considerazione alcuni specifici passi dell'opera. Certamente è importante ribadire che, dato lo stato estremamente lacunoso del testo, risulta arduo trarre conclusioni che siano valide in senso assoluto. Ciononostante, i frammenti in nostro possesso permettono di fare alcune osservazioni.

\section{L'INTERESSE PER LE IMBOSCATE}

È stato già sottolineato in passato un particolare interesse da parte dell'autore delle Elleniche di Ossirinco nel descrivere imboscate e tranelli ${ }^{28}$.

È noto che la narrazione della vittoria riportata da Agesilao ai danni di un esercito persiano nei pressi di Sardi, o come la definiscono alcuni la battaglia del fiume Pattolo ${ }^{29}$, nell'estate del 395 contenuta nell'undicesimo capitolo delle Elleniche di Ossirinco si discosta in alcu-

28 Oltre a quelli esaminati in questo paragrafo, altri stratagemmi sono presenti nella narrazione della battaglia di Nozio (Hell. Oxy. 4) e in quella degli eventi che videro coinvolto Conone a Rodi (Hell. Oxy. 15 e 20); cfr. McKechnie - Kern 1988, 16 e n. 1.

29 E. g. Dillery 1995, 109.

30 Diod. XIV.80.1-4. Cfr. anche Paus. 3.9.6, che colloca la battaglia Ėv "E $\rho \mu o v$ $\pi \varepsilon \delta i ́ \omega$. 
ni dettagli dal racconto della battaglia riportato da Diodoro ${ }^{30}$, ma soprattutto differisce in più punti in maniera sostanziale dall'altro resoconto contemporaneo agli eventi a nostra disposizione, quello di Senofonte nelle Elleniche ${ }^{31}$ e nell'Agesilao ${ }^{32}$. Tali discrepanze sono apparse spesso inconciliabili, nonostante alcuni tentativi anche recenti da parte degli studiosi di armonizzare le varie versioni ${ }^{33}$, e hanno condotto i moderni a dividersi tra quanti prediligono la versione senofontea $^{34}$ e coloro che, invece, danno maggior credito all'Anonimo e a Diodoro $^{35}$. Se da un lato, infatti, le diversità tra il resoconto diodoreo e quello dell'autore delle Elleniche di Ossirinco non sembrano così marcate $^{36}$, ben più netta è la distanza fra la narrazione di quest'ultimo e quella fatta da Senofonte ${ }^{37}$.

In particolare, Diodoro e le Elleniche di Ossirinco riferiscono di un'imboscata tesa da un manipolo di uomini di Agesilao al comando di un certo Senocle, episodio che è del tutto assente dalla narrazione delle Elleniche e dell'Agesilao senofontei.

Secondo l'Anonimo, il re spartano avrebbe appunto selezionato un certo numero di opliti e armati alla leggera e avrebbe posto al loro comando uno spartiata di nome Senocle, ordinando loro di attaccare i Persiani nel momento opportuno. Il racconto di Diodoro segue sostanzialmente quello delle Elleniche, con alcune minime discrepanze. In primo luogo, nello storico siceliota leggiamo il numero di combattenti che formavano il manipolo, millequattrocento; tuttavia egli parla genericamente di "soldati" ( $\sigma \tau \rho \alpha \tau \iota \omega ́ \tau \alpha \iota)$, senza distinguere fra le diverse

31 Xen. Hell. III.4.20-25.

32 Xen Ages. 1.28-33. Al medesimo scontro fa riferimento anche Plut. Ages. 10.1-4, che segue sostanzialmente il racconto senofonteo con un'unica netta differenza, ovvero la presenza di Tissaferne direttamente sul campo di battaglia; cfr. Bruce 1967, 151-152.Vedi anche Nepos Ages. 3.4-5; Polyaen. II, 1, 9; Front., Strat. I 8, 12.

33 Lotz 2016.

34 Come ad esempio Anderson 1974 e Gray 1979.

35 Dugas 1910; Bruce 1967, 150-156; Nellen 1972.

36 Nello specifico diverse sono le cifre relative ai caduti tra le fila dei persiani, seicento secondo l'Anonimo, seimila secondo Diodoro. Inoltre, Diodoro riporta l'episodio dell'incursione nella tenuta di Tissaferne, che non sembra possibile individuare all'interno delle Elleniche di Ossirinco.

37 Un punto fondamentale su cui i moderni si sono divisi è dove esattamente si sia combattuta la battaglia. Cfr. Botha 1988, 71-80; DeVoto 1988. Per le differenze tra le due versioni, cfr. Bruce 1967, 152. 
tipologie di fanti, come invece fa lo storico di Ossirinco parlando di cinquecento $\psi i \lambda \mathrm{oi}^{38}$ e di un numero di opliti per noi non ricostruibile a causa di una lacuna del papiro. In aggiunta, da Diodoro veniamo a sapere che questi uomini furono collocati in un'area boscosa. Le due testimonianze non sono certo incompatibili l'una con l'altra, e anzi la diversa maniera in cui le due fonti descrivono la composizione dell'armata di Senocle sembra deporre a favore di una maggiore attenzione ai dettagli da parte dell'autore più antico.

Secondo le Elleniche di Ossirinco, inoltre, la sequenza di attacco è la seguente: in primo luogo Senocle con i suoi uomini lascia la posizione coperta e attacca i Persiani, causando il panico, poi interviene Agesilao con il resto dell'esercito, costringendo Tissaferne a ritirarsi verso Sardi e a lasciare sul campo seicento caduti. In Diodoro l'ordine di attacco è invertito: Agesilao, una volta superato il punto in cui erano posizionati gli uomini pronti all'imboscata, fa invertire improvvisamente la marcia ai suoi e assalta i Persiani; solo a questo punto Senocle con i suoi uomini si lancia nella mischia. Il numero di vittime tra le fila dei persiani secondo lo storico siceliota è di seimila ${ }^{39}$. Se quest'ultima differenza è ascrivibile a un errore del copista, la diversa successione degli eventi tra le due opere può essere attribuibile a una distrazione da parte di Diodoro nell'epitomare la narrazione di Eforo o addirittura di Eforo stesso nel suo testo originale ${ }^{40}$.

Ad ogni modo le ricostruzioni della battaglia proposte dalle due fonti sono sostanzialmente compatibili ed è probabile che Eforo abbia utilizzato come fonte per questo passo le Elleniche di Ossirinco.

Ben più marcata è la distanza tra il resoconto dell'anonimo e quello di Senofonte. Secondo quest'ultimo, infatti, Agesilao mosse da Efeso in direzione di Sardi senza incontrare nemici. Il quarto giorno la cavalleria nemica apparve e, attraversando il fiume Pattolo, attaccò i Greci che si erano dati al saccheggio. Il re rispose ordinando ai suoi cavalieri di andare in aiuto di quanti erano stati attaccati. Agesilao stesso, volendo cogliere l'opportunità offerta dal fatto che sul campo non era presente la fanteria persiana, schierò la sua fanteria comandando agli opliti

38 Il numero è parzialmente integrato.

39 Diod. XIV, 80.

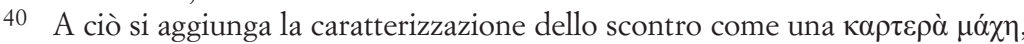
laddove nelle Elleniche di Ossirinco si dice che i Persiani offrirono scarsissima resistenza. Tale sfumatura ben si addice a uno stile storiografico retorico come quello dello storico di Cuma; cfr. Bruce 1967, 150-151. 
delle prime dieci classi d'età di muoversi a passo rapido contro la linea nemica, lasciando inoltre che i peltasti facessero lo stesso a ritmo ancora più veloce; infine diede ordine anche ai cavalieri di lanciarsi all'attacco. I Persiani offrirono un'iniziale resistenza, ma poi furono costretti a ripiegare e alcuni di loro furono uccisi ${ }^{41}$.

Di imboscate nessuna traccia. In tutto questo, inoltre, Tissaferne, che secondo lo storico di Ossirinco era al comando delle sue truppe, stando a Senofonte si trovava a Sardi e questo gli costò il titolo di satrapo e, in ultima istanza, la vita ${ }^{42}$.

Senocle, inoltre, che secondo le Elleniche di Ossirinco e Diodoro era al comando del manipolo di uomini incaricato da Agesilao dell'esecuzione dell'agguato, viene menzionato da Senofonte pochi paragrafi prima della descrizione dello scontro nei pressi del fiume Pattolo nel novero dei trenta spartiati che giunsero in Asia Minore all'inizio del nuovo anno al seguito di Erippida, che aveva preso il posto di Lisandro al seguito della spedizione. A lui e a un altro uomo di cui non viene rivelato il nome Agesilao assegnò il comando della cavalleria.

La distanza tra i due resoconti è talmente netta, che alcuni studiosi sono arrivati a concludere che i due autori stanno descrivendo due distinte battaglie che avrebbero avuto luogo nei pressi di Sardi ${ }^{43}$, ipotesi respinta da altri sulla base del fatto che, se Agesilao avesse riportato due vittorie, difficilmente Senofonte avrebbe omesso di riferirne una ${ }^{44}$. Tra chi, invece, ha cercato di conciliare le due narrazioni ${ }^{45}$, alcuni hanno messo fortemente in dubbio l'attendibilità di Senofonte a tutto favore del racconto dell'anonimo ${ }^{46}$. Dal canto di quanti propendono a favore di Senofonte ${ }^{47}$, invece, si è sottolineata una certa convenzionalità nella descrizione dell'imboscata da parte dell'autore delle Elleniche di

41 Xen., Hell. III, 4, 20-24.

42 Xen., Hell. III, 4, 25.

43 E.g. Thomas 2009, ix-lxvi; Flower 2017, 313-314.

44 E.g. Cornelius 1933 e Cawkwell 1968. Bruce 1967, 152-153 suggerisce che Senofonte, che pure aveva preso parte alla campagna in Asia Minore, potrebbe non aver seguito l'esercito in marcia in direzione di Sardi, rimanendo invece a Efeso e, quindi, basando il proprio racconto di questi eventi su testimonianze incerte e omettendo di riferire quella che, in fin dei conti, era poco più di una schermaglia.

45 Come ha fatto DeVoto 1988.

46 E.g. Dugas 1910; Cawkwell 1979, 405-406.

47 Come ad esempio Anderson 1974, 27-53; Wylie 1992.

48 E.g. Walker 1921, 128; Gray 1979, 195-196. 


\section{Ossirinco $^{48}$}

Non è lo scopo di questo contributo schierarsi dall'una o dall'altra parte nel dibattito, in ultima istanza senza fine, su quale delle due versioni sia da preferire, quanto piuttosto cercare di capire se l'autore delle Elleniche di Ossirinco possa essere considerato un valido storico militare. In questo senso è importante ricordare che, come Senofonte, anche l'anonimo può avere selezionato le informazioni in base ai suoi interessi personali. Senofonte, infatti, si concentra sugli eventi che coinvolgono la cavalleria ${ }^{49}$, il che ben si spiega con l'importanza che per lo storico amico di Agesilao aveva questa arma negli eserciti, tanto da dedicare un trattatello formativo destinato al comandante di cavalleria. Così anche l'anonimo di Ossirinco, che peraltro non era presente durante la spedizione e dunque si trovò a lavorare sulla base di resoconti, può avere selezionato il materiale in base a un suo possibile interesse per gli agguati e per una maniera di combattere più astuta.

Lo stile semplice e diretto adottato dallo storico di Ossirinco per narrare l'imboscata ordita da Agesilao e messa in atto da Senocle, però, non può essere considerato elemento valido per classificare questa descrizione come convenzionale. La medesima netta sequenzialità adottata nel riferire di questo episodio si ritrova anche nel resoconto dei due agguati menzionati in precedenza che videro coinvolto nuovamente l'esercito di Agesilao, una volta come vittima, l'altra come esecutore, questa volta in Misia ${ }^{50}$.

Come detto, mentre l'esercito lacedemone stava affrontando uno stretto passaggio nei pressi del Monte Olimpo, la retroguardia fu attaccata dai Misi, che uccisero una cinquantina di uomini. In risposta, Agesilao comandò agli uomini di Dercillida di preparare un agguato ${ }^{51}$, mentre il re con il resto dell'esercito iniziò a compiere una manovra diversiva, che spinse i Misi impegnati nell'attacco a cadere in trappola, perdendo più di centotrenta combattenti. Che questa descrizione sia

49 Nella stessa sezione del libro IV delle Elleniche, Senofonte descrive con dovizia di particolari uno scontro tra cavallerie nei pressi di Dascilio, dove gli uomini

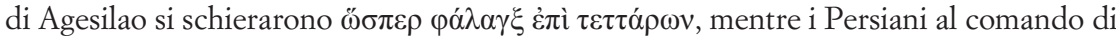

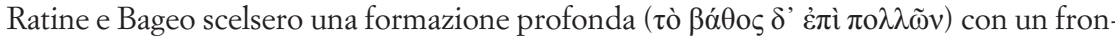
te di dodici cavalieri; cfr. Xen., Hell. III, 4, 12-14.

50 Hell. Oxy. 21, 2.

51 Sull'identità di questi uomini, cfr. McKechnie - Kern 1988, 180. 
convenzionale è opinione che già Iain Bruce, nel suo commento all'opera, respingeva $a^{52}$. La somiglianza tra questa imboscata e quella tesa a Tissaferne, che lo stesso Bruce notava, non è da attribuire a un espediente narrativo dell'autore, quanto piuttosto alla prassi tattica di Agesilao, notando anche come i due episodi siano simili, ma non uguali. Si segnala, invece, da parte dell'autore precisione e metodicità nel riportare gli eventi in uno stile certamente non retorico, ma asciutto e disadorno.

Tale accuratezza e sistematicità nel riferire di alcuni aspetti degli eventi bellici si ritrova, ad esempio, nella descrizione dei movimenti di eserciti e di contingenti più piccoli sia per terra che per mare.

\section{LA CURA NEL RIFERIRE GLI SPOSTAMENTI}

Questa dinamica emerge chiaramente nel resoconto degli eventi che fecero seguito alla vittoria greca contro l'esercito di Tissaferne nei pressi di Sardi. Senofonte nelle Elleniche muove la sua attenzione dalla cattura del campo persiano all'omicidio di Tissaferne ${ }^{53}$, facendo solo un accenno quasi accidentale alcuni paragrafi dopo al fatto che il re spartano aveva spostato l'esercito in Frigia ${ }^{54}$. Nell'Agesilao, addirittura, egli a malapena menziona il fatto che i Greci si erano dati a devastare la piana di Sardi dopo la battaglia ${ }^{55}$.

Al contrario le Elleniche di Ossirinco dedicano maggiore spazio ai movimenti compiuti da Agesilao e dal suo esercito: il re sarebbe rimasto sul campo di battaglia per tre giorni al fine di compiere tutti i riti necessari ed erigere il trofeo; poi avrebbe marciato attraverso la Lidia e le montagne che separano questa regione dalla Grande Frigia, senza tenere i suoi uomini in formazione e lasciandoli piuttosto liberi di saccheggiare; infine egli sarebbe arrivato alla piana del fiume Meandro, vicino a Celene, ma, dal momento che i presagi gli erano avversi, decise di ritirarsi ${ }^{56}$. Purtroppo, da questo punto in avanti il testo è fortemente dan-

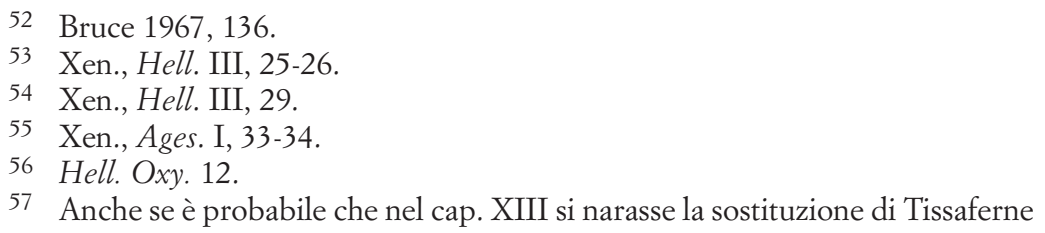


neggiato e pertanto non possiamo seguire gli sviluppi della narrazione ${ }^{57}$.

La medesima cura nel riportare i movimenti dell'esercito di Agesilao si riscontra nei capitoli 21 e 22 dell'opera, laddove lo storico registra in successione e in uno stile essenziale e quasi telegrafico due falliti assalti agli insediamenti frigi di Leonton Kephalai ${ }^{58}$ e di Gordio, seguiti da una marcia attraverso la Paflagonia e lungo il fiume Sangario. L'esercito, poi, avrebbe raggiunto Kios in Misia, tornando in seguito nuovamente in Frigia, dove avrebbe tentato e ancora una volta non sarebbe riuscito ad assaltare il bastione di Miletou Teichos ${ }^{59}$. Infine, costeggiando il Fiume Rindaco, Agesilao sarebbe giunto a Dascilio, laddove Farnabazo teneva tutti i suoi tesori ${ }^{60}$.

Nelle Elleniche Senofonte si occupa di questi eventi nei primi diciassette paragrafi del primo capitolo del libro quattro. Tuttavia, egli dedica molto spazio ai dialoghi e alla descrizione dei luoghi, come ad esempio di Dascilio. Dopo aver introdotto il personaggio di Titrauste e aver annotato la tregua tra questi e Agesilao ${ }^{61}$, lo storico prosegue la narrazione riportando che il re spartano, giunto all'inizio dell'autunno in Frigia, devastò la regione e conquistò città, alcune con la forza, altre ottenendone la sottomissione volontaria ${ }^{62}$. Manca qualsivoglia riferimento a un'azione specifica.

A questo punto Senofonte inizia a descrivere le trattative matrimoniali condotte tra Spitridate e Otys, re dei Paflagoni ${ }^{63}$, nelle quali Agesilao agì da mediatore, e riferisce del vero e proprio incontro tra $\mathrm{i}$ re, dedicando molti paragrafi al presunto dialogo tra i due ${ }^{64}$. Solo al paragrafo quindicesimo lo storico annota che l'esercito lacedemone era giunto a Dascilio, che, come detto, egli delinea in maniera particolareg-

con Titrauste e l'uccisione del primo; cfr. McKechnie - Kern 1988, 148. Sulla rotta seguita dall'esercito di Agesilao, cfr. Anderson 1974, 33-41.

58 Su cui cfr. Berndt Ersöz 2018, 23-53.

59 Sull'identificazione di questo sito cfr. Bruce 1967, 145-146.

60 Hell. Oxy. 21-22.

61 Cfr. anche Diod. XIV, 80, 8, il quale parla di una tregua della durata di sei mesi. Nel testo preservato delle Elleniche di Ossirinco. non v'è traccia di questa tregua, anche se è possibile che un riferimento fosse presente nella parte perduta del testo; cfr. McKechnie - Kern 1988, 177.

62 Xen., Hell. IV, 1, 1-2.

63 Chiamato Cotys in Xen., Ages. 3, 4 e Gyes in Hell. Oxy. 22, 1.

64 Xen., Hell. IV, 1, 2-15

65 Xen., Hell. IV, 1, 15-17. 
giata come luogo dove si trovava il palazzo di Farnabazo ${ }^{65}$. Anche in questo caso, dunque, le Elleniche di Ossirinco forniscono informazioni supplementari rispetto alla narrazione di Senofonte con un approccio sistematico e metodico.

Questa caratteristica, tuttavia, non si manifesta soltanto nel ricostruire il percorso seguito dall'esercito di Agesilao. La stessa precisione si riscontra, ad esempio, nel racconto della spedizione condotta dalla Lega beotica in Focide, sempre nel $395^{66}$. Anche in questo caso nella narrazione non v'è spazio per digressioni e verbosità. Una volta penetrato nella regione nemica, l'esercito beotico si diede a devastare il territorio di Parapotamio, Daulia e Fanoteo, con risultati alquanto parziali. Vano, infatti, fu il tentativo dei Beoti di catturare l'insediamento di Daulia, respinti con perdite: essi riuscirono solo a occupare con la forza i sobborghi di Fanoteo. In seguito, si spinsero all'interno del territorio focese, facendo incursioni nell'area di Elate ${ }^{67}$ e di Pedieo. Giunti nella regione di Iampoli, cercarono di assaltare la città, descritta dall'anonimo come un insediamento fortificato in maniera adeguata ( $\chi \omega$ píov $\dot{\varepsilon} \pi \imath \varepsilon \kappa \tilde{c} \varsigma$ i $َ \chi \cup \rho o ́ v)$, ma non riuscirono nell'intento e anzi persero ottanta uomini. A questo punto decisero di ritirarsi e tornare in Beozia.

Di questo in Senofonte non v'è traccia: lo storico si limita a dire che, su pressione della fazione di Androclide, i Tebani invasero la Focide, saccheggiandola ${ }^{68}$. In tutti e tre i casi riportati, dunque, l'anonimo di Ossirinco fornisce informazioni sugli spostamenti di un esercito che non sono presenti in Senofonte.

La precisione dell'autore delle Elleniche di Ossirinco nel fornire una dettagliata ricostruzione degli eventi era già stata attribuita da Christopher Dugas quasi un secolo fa all'uso dei racconti di testimoni oculari $^{69}$. Inoltre, è riconosciuto dai moderni come la ricostruzione delle battaglie da parte dell'anonimo sia con tutta probabilità basata su fonti ben informate, ovvero rapporti ufficiali scritti dai generali, ma anche dai diari composti da semplici soldati ${ }^{70}$. Queste erano precisamente le

\footnotetext{
66 Hell. Oxy. 18, 5.

67 Sull'importanza strategica di Elatea, cfr. Bruce 1967, 122.

68 Xen., Hell. III, 5, 4.

69 Dugas 1910, 92.

70 Bruce 1967, 7-8.
} 
tipologie di documenti in cui era possibile trovare i dettagli descritti in maniera minuziosa e con quello stile asciutto e diretto che si ritrova anche nell'anonimo. Un'accuratezza che, d'altro canto, l'autore mostra anche nel descrivere altri elementi non legati alla sfera militare, come ad esempio dimostrato nel quadro fornito relativo al funzionamento della Lega beotica ${ }^{71}$.

Bisogna però ricordare che, per quanto questo approccio permetta a noi di avere molte utili informazioni per la ricostruzione degli eventi, che spesso non è possibile trovare in Senofonte, il semplice riportare una serie di eventi facendo un collage delle fonti a propria disposizione non è sufficiente a caratterizzare l'autore delle Elleniche di Ossirinco come uno storico militare. Come detto, infatti, uno storico militare non si limita alla semplice narrazione di battaglie o scontri di minore entità, ma aggiunge analisi e approfondimenti su temi specifici, da un punto di vista sincronico e diacronico.

\section{L'ANALISI APPROFONDITA DEGLI ASPETTI ECONOMICI}

Ora, l'unico esempio a nostra disposizione di indagine approfondita di una questione legata alla sfera della guerra da parte dell'anonimo è presente nel diciannovesimo capitolo, laddove si discutono i limiti dell'approccio adottato dal Gran Re nel pagare i suoi soldati come preludio all'ammutinamento delle truppe persiane guidate da Conone presso Cauno, innescato precisamente da un ritardo di molti mesi nel pagamento dei salari. In questo punto l'autore sembra esprimere una disamina personale della questione, visto che dichiara che l'unico responsabile di questa mancata retribuzione era Artaserse ( $\tau$ ov́ $\tau \omega v \delta \grave{\varepsilon}$ $\beta \alpha \sigma i \lambda \varepsilon \dot{\varsigma} \varsigma \alpha$ i̊ıı́s $\dot{\varepsilon} \sigma \tau \imath v)$, dal momento che egli, ogniqualvolta decideva di dichiarare guerra, inviava una somma ridotta di denaro (ỏ $\lambda \dot{i} \gamma \alpha \chi \rho \eta \dot{\mu} \mu \alpha \tau \alpha)$ al principio a quelli che erano in comando dell'esercito e non si curava

71 Steui cfr. Lanzillotta 2001, 119-127.

72 Hell. Oxy. 19, 2. Come già sottolineava Breitenbach 1970, 399, questa porzione di testo può essere considerato un excursus sulle difficoltà generalizzate incontrate dalle truppe mercenarie greche al servizio del Gran Re per quanto riguarda la retribuzione del servizio, ricollegandosi alla guerra deceleica; cfr. anche Bruce 1967, 125. Secondo Accame 1978, 148-149, l'inizio del mancato pagamento sarebbe da collocare al momento del raduno della flotta persiana o comunque alla fine del 397, dal momento 
di quanto accadeva in seguito ${ }^{72}$.

Questo tipo di atteggiamento da parte dei Persiani era già stato rilevato da Tucidide, il quale faceva menzione della riduzione della paga versata ai Peloponnesiaci da parte di Tissaferne da una dracma attica a tre oboli, riduzione consigliata da Alcibiade il quale, in quel momento, puntava a danneggiare gli Spartani ${ }^{73}$, una riduzione che suscitò forte malcontento tra i soldati ${ }^{74}$. Tucidide, tuttavia, attribuisce questo episodio all'influenza esercitata su Tissaferne da Alcibiade, che lo storico ateniese descrive addirittura come maestro di ogni argomento del satrapo

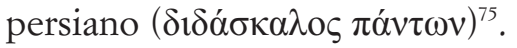

Al contrario, invece, l'autore delle Elleniche di Ossirinco dimostra di avere una conoscenza del fenomeno sufficiente a permettergli di fornire una spiegazione plausibile: dovendo raccontare la vicenda dell'ammutinamento a Cauno ${ }^{76}$ e sapendo che esso era dovuto a un difetto nel versamento della paga agli uomini comandati da Conone, egli fornisce una analisi delle cause strutturali che stavano alla base dell'episodio. Non, dunque, la nefasta influenza di un esule ateniese, come nel caso di Tissaferne, ma un atteggiamento poco attento e quasi disinteressato da attribuirsi direttamente al Gran Re e ai suoi delegati ${ }^{77}$.

D'altro canto, questo giudizio riecheggia quanto detto anche da Isocrate in merito a questo episodio. L'oratore ateniese, infatti, nel Panegirico ricordava, quale esempio di estrema lentezza nelle azioni messe in atto da parte del Gran Re (

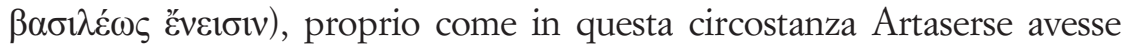
lasciato senza paga gli uomini di Conone per addirittura quindici mesi.

Stando all'anonimo, la questione si risolse con l'invio, da parte di

che l'anno prima Farnabazo aveva ricevuto cinquecento talenti dal Gran Re per armare la flotta; cfr. Diod. XIV, 39, 1. March 1997, 265-266, ha suggerito che il mancato pagamento dei soldati di Conone sia invece da attribuire a una precisa strategia di Artaserse, maggiormente preoccupato dalla minaccia rappresentata dall'esercito in marcia alla guida di Agesilao e quindi più propenso a finanziare le truppe di terra, a detrimento della flotta.

73 Thuc. VIII, 45, 1-2.

74 Thuc. VIII, 83, 2-3 e 99.

75 Thuc. VIII, 45, 2.

76 Riguardo a questo ammutinamento, l'unica altra fonte disponibile è Iust. VI, 2, 11, il quale si limita alla semplice constatazione dell'evento. L'episodio manca del tutto in Senofonte.

77 Isocr., Paneg. 141-142. 
Titrauste, di una somma pari a duecentocinquanta talenti d'argento prelevati direttamente dai beni privati di Tissaferne. Lo stesso Titrauste, prima di spostarsi da Sardi verso Susa per incontrare il Gran Re, avrebbe lasciato il comando delle manovre militari ad Arieo e Pasiferne, con oro ed argento per un ammontare complessivo di settecento talenti $\mathrm{i}^{78}$.

Quanto detto, dunque, testimonia come l'autore delle Elleniche di Ossirinco sia interessato ed esprima opinioni personali derivanti, con tutta probabilità, dall'esame di informazioni in suo possesso per quanto riguarda la sfera economica quando questa si sovrappone a quella militare, e questo è precisamente uno degli elementi che rientrano nella definizione ampia di "storico militare" che abbiamo individuato in precedenza.

Ciononostante, l'interesse per le questioni economiche non si limita a quando queste hanno a che fare con l'esercito, ma è una delle caratteristiche trasversali dell'opera, come dimostrato da quanto l'autore dice al capitolo diciassette, laddove dedica un excursus al miglioramento della situazione economica, accanto a quella demografica, a Tebe nell'ultimo trentennio del V secolo, allorché i Tebani riuscirono a trarre profitto dall'alleanza con gli Spartani e dall'occupazione da parte di questi ultimi della fortezza di Decelea, a detrimento degli Ateniesi ${ }^{79}$.

Inoltre, una certa attenzione per i flussi di denaro e per le motivazioni economiche, in questo caso dietro alle scelte politiche, può essere individuata anche nel settimo capitolo, laddove l'anonimo afferma che all'inizio della guerra di Corinto quelli degli Ateniesi che facevano pressione affinché l'assemblea votasse per dichiarare guerra agli Spartani erano animati da concrete motivazioni di tipo economico, ovvero il desiderio riempire le proprie tasche con le ricchezze del tesoro pubblico ${ }^{80}$.

\section{CONCLUSIONI}

In conclusione, dunque, l'autore delle Elleniche di Ossirinco appare come uno storico metodico e piuttosto sistematico nel racco-

78 Hell. Oxy. 19, 3. Per una stima dei costi di mantenimento degli equipaggi di Conone, cfr. McKechnie - Kern 1988, 172. Secondo Pritchett 1971, 24-25, questo andamento irregolare nel versamento della paga ai mercenari rispondeva a una precisa strategia da parte del Re, il quale puntava a tenere sulle spine i soldati limitando le spese.

79 Hell. Oxy. 17, 3-5.

80 Hell. Oxy. 7, 2. 
gliere e presentare i fatti in uno stile lineare e piuttosto essenziale. Questa caratteristica non si limita alla narrazione di eventi militari, ma si ritrova in maniera diffusa nell'intera opera. Egli poteva fare uso di fonti piuttosto accurate, fossero esse testimonianze orali o scritte di testimoni o rapporti ufficiali delle operazioni militari. L'autore integra queste informazioni all'interno del suo racconto con un occhio attento, ad esempio, a registrare i movimenti degli eserciti o il numero dei caduti di alcuni scontri.

A ciò si aggiunga che spesso l'autore aggiunge dettagli che nell'altra fonte storica contemporanea agli eventi, Senofonte, sono assenti, quasi a testimoniare un desiderio di integrare alcuni aspetti mancanti.

Peraltro, l'autore non si limita alla mera narrazione degli eventi, ma aggiunge anche osservazioni che testimoniano il suo interesse per svariati argomenti, come nel caso delle dinamiche economiche relative agli episodi bellici. Ciononostante, l'analisi fornita riguardo i problemi connessi con la maniera persiana di pagare i soldati è l'unico caso in nostro possesso in cui l'anonimo inserisce considerazioni personali su un argomento specifico che risulta collegato con la sfera militare.

Certo, non sarebbe prudente trarre conclusioni definite sull'operato dell'autore delle Elleniche di Ossirinco, dal momento che in nostro possesso abbiamo solo una parte dell'opera. Tuttavia, l'affermazione di Cartledge, secondo il quale l'anonimo agisce come un valido storico militare semplicemente sulla base del fatto che la sua narrazione, a differenza di quella di Senofonte, è priva di secondi fini, perché si concentra esclusivamente su informazioni rilevanti per permettere al lettore di avere un'adeguata comprensione dell'accaduto e perché fa tutto ciò in uno stile per nulla retorico, risulta quantomeno poco appropriata se pensiamo al significato che la definizione "storico militare" ha per noi oggi. Risulta più prudente, invece, considerare le Elleniche di Ossirinco un'opera composta da uno storico metodico e sotto molti aspetti puntiglioso, con una curiosità vivace per svariati aspetti, dalla sfera politica e istituzionale a quella economica e, per quanto riguarda quella militare, per le imboscate e gli spostamenti degli eserciti, che egli cerca di ricostruire in maniera abbastanza sistematica, fornendo talvolta opinioni personali. Uno storico sicuramente poliedrico, per quanto forse non sempre la lettura più gradevole da un punto di vista stilistico. 


\section{BIBLIOGRAFIA}

Accame 1978. S. Accame, Ricerche sulle Elleniche di Ossirinco, MGR 6 (1978), 125-183. Anderson 1974. J.K. Anderson, The battle of Sardis in 395 B.C., CSCA 7 (1974), 27-53. Bearzot 1985. C. Bearzot, La constituzione beotica nella propaganda degli oligarchici ateniesi del 411, in La Beotie antique. Actes des Colloques internationaux du CNRS, Paris, Éditions du Centre national de la recherche scientifique, 1985 , 219-226.

Back 2004. J. Black, Rethinking Military History, London, Routledge, 2004.

Berndt Ersöz 2018. S. Berndt Ersöz, The Historical Geography of Phrygia: The Settlement of Leonton Kephalai, Orbis Terrarum 16 (2018), 23-53.

Bonner 1910. R.J. Bonner, The Boeotian Federal Constitution, CP 5 (1910), pp. 405-417

Botha 1988. L. Botha, The Asiatic campaign of Agesilaus. The topography of the route from Ephesus to Sardis, Acta Classica 31 (1988), 71-80.

Breitenbach 1970. H.R. Breitenbach, Hellenika Oxyrbynchia, in RE Suppl. 12 (1970), 383-426.

Bruce 1962. I.A. F. Bruce, The Political Terminology of the Oxyrbynchus Historian, Emerita 30 (1962), pp. 63-69.

Bruce 1967. I.A. F. Bruce, An historical commentary on the Hellenica Oxyrynchia, Cambridge, Cambridge University Press, 1967.

Cartledge 1987. P. Cartledge, Agesilaos and the Crisis of Sparta, Baltimore, Johns Hopkins University Press, 1987.

Cawkwell 1968. G.L. Cawkwell, The Oxyrbynchus bistorian, Classical Review 18 (1968), 288-290

Cawkwell 1979. G.L. Cawkwell, Xenophon. A History of My Times, London, Penguin Classics, 1979.

Citino 2007. R.M. Citino, Military histories old and new: A reintroduction, The American Historical Review 112 (2007), 1070-1090.

Cornelius 1933. F. Cornelius, Die Schlacht bei Sardes, Klio 26 (1933), 29-31.

DeVoto 1988. J. DeVoto, Agesilaos and Tissaphernes near Sardes in 395 B.C., Hermes 116 (1988), 41-53.

Dillery 1995. J. Dillery, Xenophon and the History of His Time, London, Routledge, 1995.

Dugas 1910. C. Dugas, Le campagne d'Agésilas en Asie Mineure, BCH 34 (1910), 58-95.

Flower 2017. M.A. Flower, Xenophon as a Historian, in M. A. Flower (ed.), The Cambridge Companion to Xenophon, Cambridge, Cambridge University Press, 2017, 301-322.

Gray 1979. V.J. Gray, Two different Approaches to the battle of Sardis in 395 B.C. Xenophon Hellenica 3.4.20-24 and Hellenica Oxyrbynchia 11(6).4-6, CSCA 12 
(1979), 183-200.

Howard et al. 1988. M. Howard et al., What is Military History?, in J. Gardiner (ed.),

What is History Today, London, Macmillan, 1988, 4-17.

Lanzillotta 2001. E. Lanzillotta, Interessi costituzionali nelle Elleniche di Ossirinco, Sileno 27 (2001), 119-127.

Lérida Lafarga 2007. R. Lérida Lafarga, Comentario bistórico de las Helénicas de Oxirrinco, Zaragoza, Institución Fernando el Católico, 2007.

Lotz 2016. H. Lotz, Die "Schlacht bei Sardeis" 395 v.Chr. Ein historiographisches Konstrukt?, Historia 65 (2016), 126-154.

March 1997. D.A. March, Konon and the Great King's fleet, 396-394, Historia 46 (1997), pp. 256-269.

McKechnie - Kern 1988. P.R. McKechnie - S.J. Kern, Hellenica Oxyrhynchia, Warminster, Aris \& Phillip Ltd., 1988.

Morillo - Pavkovic 2006. S. Morillo - M.F. Pavkovic, What Is Military History?, Malden (Mass.), Polity Press, 2006,

Mossé 2001. C. Mossé, La bataille de Notion dans la Vie d'Alcibiade de Plutarque et dans les Helleniques d'Oxyrbynchos, Sileno 27 (2001), 189-192.

Nellen 1972. D. Nellen, Zur Darstellung der Schlacht bei Sardes in den Quellen, Ancient Society 3 (1972), 45-54.

Occhipinti 2016. E. Occhipinti, The Hellenica Oxyrhynchia and Historiography: New Research Perspectives, Leiden, Brill, 2016.

Pritchett 1971. W.K. Pritchett, The Greek State at War, I, Berkeley - Los Angeles, University of California Press, 1971.

Thomas 2009. D. Thomas, Introduction, in R. Strassler (ed.), The Landmark Xenophon's Hellenika, New York, Pantheon Book, 2009.

Walker 1921. E.M. Walker, The Oxyrhynchus Historian, in J.U. Powell - E.A. Barber (eds.), New Chapters in the History of Greek Literature, Oxford, Clarendon Press, 1921, 124-133.

Wylie 1992. G. Wylie, Agesilaus and the Battle of Sardis, Klio 74 (1992), 118-130. 
